\title{
Sobre un esgrafiado con onomástica ibérica del Tossal de Cal Montblanc (Albesa, Lleida)
}

\author{
On an Iberian graffito from \\ El Tossal de Cal Montblanc \\ (Albesa, Lleida)
}

\author{
Víctor Revilla Calvo \\ Universitat de Barcelona \\ vrevilla@ub.edu \\ Javier Velaza Frías \\ Universitat de Barcelona \\ velaza@ub.edu
}

\begin{abstract}
Resumen: La excavación de un asentamiento rural cercano a Lleida ha permitido recuperar un importante contexto cerámico datado entre finales del siglo I e inicios del II d.C. El depósito incluye una amplia representación de terra sigillata y vajillas de uso culinario del periodo. El interés del repertorio reside en la diversidad y cantidad de los esgrafiados conservados. Los textos se escribieron casi exclusivamente en latín, pero incluyen algún ejemplo en griego. $\mathrm{Su}$ contenido se limita a representaciones onomásticas, aunque también se identifican indicaciones de propiedad y expresiones insultantes o jocosas. En este artículo se analiza uno de los fragmentos, perteneciente a una jarra en cerámica engobada, que parece conservar una indicación onomástica ibérica y tal vez un teónimo. Esta hipótesis conferiría un carácter votivo a la inscripción.
\end{abstract}

Palabras clave: Onomástica ibérica. Epigrafía romana. Esgrafiado.

Abstract: The excavation of a rural settlement near Lleida has allowed the recovery of an important ceramic context dating from the end of the first century to the beginning of the second century AD. The deposit includes a broad representation of terra sigillata and culinary ware of the period. However, the interest of the repertoire resides in the diversity and quantity of the conserved grafitti. The texts were written almost exclusively in Latin, but include some example in Greek. Its content is limited to onomastic representations, although indications of property and insulting or jocular expressions are also identified. This article analyzes one of the fragments, belonging to a jug in common ware, which seems to retain an Iberian onomastic indication and maybe a theonym. This hypothesis would confer a votive character to the inscription.

Key words: Iberian onomastics. Roman epigraphy. Graffito.

Recepción: 03.06.2019 | Aceptación: 06.07.2019

Proyectos: Este trabajo ha sido realizado en el marco de los proyectos FFI2015-68571-P / Grup de Recerca Consolidat LITTERA (2017SGR241) y HAR2017-85635-P / Grup de Recerca Consolidat CEIPAC (2017SGR512). 


\section{El contexto del hallazgo}

Una serie de intervenciones arqueológicas realizadas en el lugar conocido como Tossal de Cal Montblanc (Albesa, provincia de Lleida) ha permitido recuperar un importante conjunto de material cerámico de época altoimperial (fig. 1). El conjunto rellenaba una fosa asociada a un asentamiento rural cuya arquitectura y naturaleza no se han podido definir con precisión. Este núcleo se sitúa en un cruce de caminos que parece fosilizar una trama de comunicaciones más antigua. Los estratos que rellenaban la fosa, depositados en un solo momento, contenían una amplia representación de la cultura material del Alto Imperio (778 individuos), en su mayor parte cerámicas destinadas a servicio de mesa y cocina, transporte, almacenamiento e iluminación. La composición del conjunto es muy desigual: las vajillas (el 51\% del total), la cerámica común oxidada y las cerámicas de cocina local e importada están bien representadas, mientras que ánforas, lucernas o producciones locales como las cerámicas engobadas aparecen en cantidad muy reducida. En la vajilla de mesa predomina la sigillata hispánica, que supone el $91,27 \%$ de esta clase y el $47,04 \%$ del total del depósito. El resto de esta clase incluye una limitada presencia de cerámica de paredes finas, cerámica vidriada y un único individuo de terra sigillata africana A. También se recuperó una limitada cantidad de tegulae e imbrices, vidrio, elementos de hierro y fauna. Las particularidades del repertorio hacen difícil asociar el conjunto a actividades exclusivamente domésticas (avance de resultados: Marí y Revilla 2018).

Fig. 1.

Localización geográfica del Tossal de Cal Montblanc (composición: R. Álvarez Arza-UB).

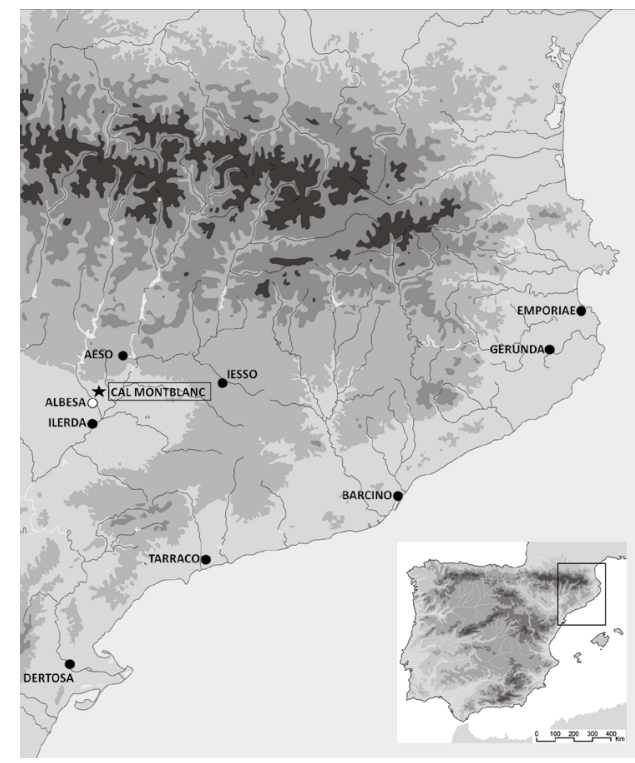


Por otro lado, la composición del conjunto es muy homogénea, ya que apenas incluye material residual, con excepción de algunos fragmentos, muy escasos, de cronología muy antigua (campaniense A y cerámica ibérica o sigillata itálica) y de las décadas centrales del s. I d.C. El momento de relleno de la fosa se sitúa en el primer cuarto/tercio del siglo II d.C., quizá en un momento más cercano al cambio de siglo (Marí y Revilla 2018, 222; para la cronología, 236).

Un cierto número de vasos cerámicos sirvió como soporte para grabar textos más o menos elaborados. Se ha recuperado un total de 144 grafitos, en su mayor parte grabados después de la cocción (Marí y Revilla 2018, 228 y ss., y figuras 10-13). En general, cada vaso muestra un único grafito, aunque se han identificado también algunas combinaciones de texto y figuras o signos. La mayoría de los grafitos aparece sobre recipientes de sigillata hispánica (66 ejemplares) y cerámica común (67 casos; la mayoría, sobre jarras). Otros aparecen en cerámica engobada (2), cerámica culinaria local (5), lucerna (1) y ánfora (1). Se ha recuperado, igualmente, un grafito sobre hueso. El repertorio es muy variado e incluye muestras complejas de escritura, motivos vegetales (palmas aisladas o entrecruzadas, hojas de hiedra) y signos más o menos complejos, como cruces, aspas y simples trazos (aunque la fragmentación impide precisar si algunos trazos formarían parte de un grafito más complejo). El aspecto más destacable de este conjunto es la presencia de onomástica, en general latina ${ }^{1}$ o griega, ${ }^{2}$ y de expresiones insultantes. ${ }^{3}$ Junto a ellos, hay también un texto llamativo por cuanto incluye onomástica paleohispánica, que es justamente el que constituye el objeto de estas páginas.

\section{El esgrafiado}

El texto (figuras 2 y 3 ) fue grabado en la mitad superior de un recipiente cerrado, muy probablemente una jarra monoansada (Marí y Revilla 2018, 233, figs. 10 y 12, $\mathrm{n}^{\circ} 7$ ). El fragmento conservaba un engobe espeso, bien aplicado y de coloración rosada, en su superficie exterior. Dadas sus características, el recipiente puede atribuirse a las producciones de cerámica revestidas de engobe producidas en el valle del Ebro entre el último siglo de la República y los prime-

$1 \operatorname{Mod}(e s t i$, -ini, -ae, -inae), Felic(is), Vit<u>li, Terti, Suc(c)e[ssi], Fortunationis, Marí y Revilla 2018, 228, no 1-3, 233, nº 10 y 15-17.

2 Eup(h)ro/sines, 'Аркос, Agat(h)emeri, Marí y Revilla 2018, 228, no 4-5 у 233, no 8.

3 Cin(a)ede, [ci]n(a)edus, Marí y Revilla 2018, 233, no 11-12. 
ros siglos del Imperio. Estas producciones, inspiradas en la tradición artesanal indígena, aparecen con frecuencia en contextos de entre los siglos I y III d.C. en ciudades como Ilerda y en numerosos contextos rurales (Morán y Payà 2007).

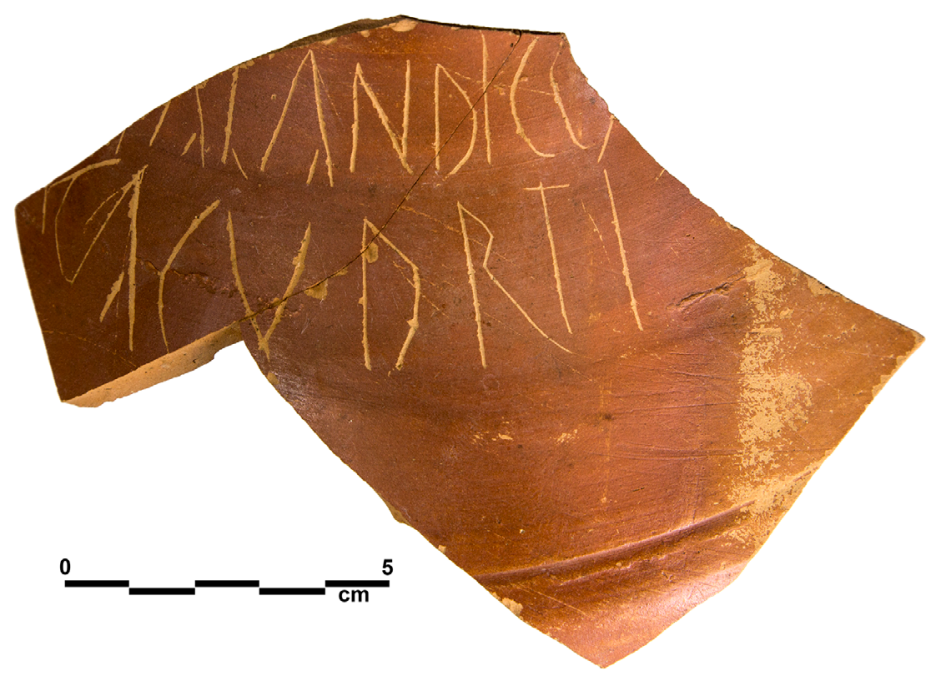

Fig. 2.

Esgrafiado sobre cerámica común engobada (foto: R. Álvarez Arza).

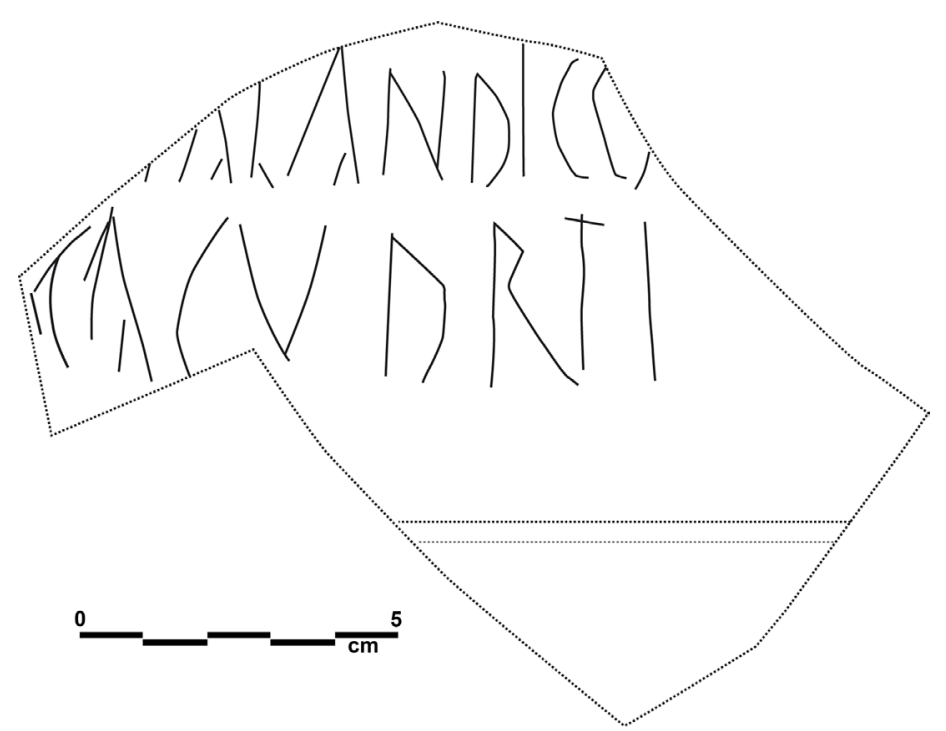

Fig. 3.

Dibujo del texto y del fragmento cerámico (dibujo: R. Álvarez Arza). 
El esgrafiado fue llevado a cabo con un instrumento de punta seca en un lugar particularmente visible del vaso y con posterioridad a su cocción. Los trazos de los signos son irregulares: especialmente la primera C de 1.2 muestra claramente los restos de vacilaciones a la hora de trazar la letra; la letra O del. 1 no cierra en su parte inferior, como tampoco la de 1. 2, cuyo ductus es todavía más deficiente (se inicia con un trazo recto vertical a partir del cual se lanzan tres trazos también rectos que intentan remedar la curvatura normal de la letra). La letra A muestra un trazo transversal muy corto y paralelo al opuesto; la L tiene también el trazo horizontal corto y ligeramente inclinado hacia abajo; la R, también muy irregular, no cierra el óculo. En general, el aspecto del esgrafiado es, pues, descuidado, aunque por tal hayamos de entender más una cierta negligencia en su trazado que una impericia por parte de su autor.

El texto se dispone en dos líneas, trazadas por una misma mano. La línea superior está claramente incompleta por su inicio, donde se conserva la parte inferior de un trazo vertical cuya restitución, como diremos a continuación, es muy problemática; sin embargo, es posible que el texto esté completo en su parte final. De la misma manera, el texto de la l. 2 podría estar completo, tanto por su parte inicial (por argumentos onomásticos que explicaremos a continuación) como final (donde, tras la letra I, hay un espacio vacío suficiente como para que se conservase al menos parte de una eventual letra siguiente).

La lectura del esgrafiado no ofrece problemas más allá de la mencionada mutilación al comienzo de la l. 1 y puede defenderse la siguiente:

$[---?]+$ alandico

Cacuorti

\section{Comentario epigráfico y onomástico}

Hasta donde entendemos, las dos líneas componen un único texto compuesto por dos palabras. De ellas, la más transparente es sin duda la de la 1 . 2, Cacuorti, que permite ser analizada como un nombre personal ibérico de formación bimembre. El primero de sus formantes lo conocemos, principalmente, por el nombre del Bagarensis del bronce de Áscoli Cacususin Chadar f(ilius), pero también probablemente por la forma kaku de Mas de Moreno (Foz Calanda, BDH TE.9.11) ${ }^{4}$ y quizás también por la forma kirkukaku (Roques de Sant Formatge, Seròs, D.17.1, aunque aquí la lectura es dudosa).

4 Citamos aquí las inscripciones ibéricas según $M L H$ o, en su defecto, según el Banco de Datos Hesperia. 
El segundo formante debe de estar relacionado con el bien conocido oŕtin / oŕdin (\$7.95), pero el hecho de que aquí no aparezca la $-n$ final es probablemente significativo: ha de recordarse, al respecto, que frente a la frecuentísima forma oŕtin / oŕdin, teníamos ya previamente algunos testimonios en los que la forma parece ser oŕti / oŕdi: así, por ejemplo, oŕti ( $B D H$ T.11.14, Tarragona), oŕti (D.15.10, Guissona), que habitualmente han sido considerados como formas abreviadas. A estos casos habría que añadir oŕtita+[ (F.9.6,1 La Punta d'Orlell, Vall d'Uixò), probablemente nombre de persona oŕti·taŕ (cuyo segundo elemento sería taŕ [\$7.115]). En este sentido, este nuevo testimonio de Tossal de Cal Montblanc vendría a abundar en la posibilidad de que exista un elemento oŕti del que oŕtin sea una variante, tal vez formada por incorporación del sufijo -(e)n, bien conocido y para el que a menudo se ha propuesto un valor equivalente al del genitivo. ${ }^{5}$

Mucho más difícil es el análisis de la palabra de 1. 1. Como se ha dicho, de su primer signo mutilado se conserva solo un breve trazo inferior vertical, lo que únicamente nos permite descartar letras de trazado curvo, como $\mathrm{O}, \mathrm{E}$, $\mathrm{D}, \mathrm{B}$, o bien otras con ángulo inferior como $\mathrm{V}$, e invitan a considerar posibilidades como $\mathrm{P}, \mathrm{T}, \mathrm{M}, \mathrm{N}$, menos probablemente $\mathrm{C}, \mathrm{R}, \mathrm{S}$. Tampoco contribuye a la interpretación de la forma su estructura fonética, donde destaca una secuencia - $n d$ - que no parece muy frecuente en ibérico (aunque recuérdense las formas asgandis (G.1.1,A-I,6 La Serreta, Alcoi), andinue+[ (G.13.1,4). Por lo demás, en el final de la palabra podría identificarse el formante onomástico -ko, bien conocido en la composición de antropónimos, pero también de teónimos (Salaeco). El inconveniente de esta identificación sería que nos dejaría en la parte inicial con un formante +alandi- sin aparentes correlatos y, lo que también resulta hasta cierto punto conflictivo, de estructura trisilábica.

Un problema ulterior es el que plantea la propia estructura del texto: si entendemos que se trata de un texto constituido por dos palabras de carácter onomástico, una solución aparentemente económica podría ser que se tratara de dos nombres personales en secuencia asindética, pero hay que reconocer que tal posibilidad no parece encontrar buenos paralelos en el resto de los ejemplares del conjunto - donde solo se menciona a un personaje en cada pieza - ni tampoco es un fenómeno habitual en la epigrafía sobre instrumentum. Así pues, convendría dejar al menos abierta una posibilidad diferente, a saber, que el primero de los dos elementos onomásticos no sea en realidad

5 La forma orti (G.1.1, A-I,1, La Serreta, Alcoi) podría corresponder a otro elemento. 
un antropónimo, sino un teónimo, y que el segundo represente al personaje que cumple una dedicatoria o un voto hacia aquel. Nos hallaríamos, de este modo, ante una inscripción de tipo votivo, función que, por lo demás, no puede descartarse para el conjunto del depósito.

Para concluir, como ya se ha dicho, la cronología de la pieza y de su contexto pueden llevarnos al primer tercio del s. II d.C., lo que nos situaría ante uno de los testimonios más tardíos conocidos de la pervivencia de la onomástica ibérica en la región.

\section{$\begin{array}{|lllllllllllll|}\text { | } & \text { B } & \text { I } & \text { B } & \text { L } & \text { I } & \text { O } & \text { G } & \text { R } & \text { A } & \text { F } & \text { I } & \text { A }\end{array}$ |}

Marí y Revilla 2018: Ll. Marí Sala y V. Revilla Calvo, "Cultura material y cultura escrita en la sociedad rural de Hispania en los siglos I-II d.C.: el conjunto cerámico y los grafitos del asentamiento de Cal Montblanc (Albesa, Lleida)", AEspA 91, 2018, 217-242.

Morán y Payá 2007: M. Morán y Payà, "La vaixella de taula engalbada de la ciutat romana d'Ilerda i el fenomen de les imitacions durant el període tardorepublicà i altimperial", en M. Roca, M. y J. Principal (eds.), Les imitacions de vaixella fina importada a la Hispania Citerior (segles I a.C.-I d.C.), Tarragona 2007, 187-234. 